\title{
Inmunogenicidad y toler ancia de una vacuna contra influenza, en una población mexicana mayor de 55 años de edad
}

\author{
0 ctavio Ayala-Montiel, MC, ,1) César Mascareñas, MC, ${ }^{(2)}$ D elfino García-Hernández, MC, ${ }^{(3)}$ \\ Jorge Rendón-Muñiz, MC, ${ }^{(1)}$ Irma López, MC, MSC, ${ }^{(4)}$ Luis Felipe Montaño, MC. ${ }^{(5)}$
}

\section{Ayala-Montiel O, Mascareñas C, García-Hernández D, Rendón-Muñiz J, López I, Montaño LF, Zenteno I, Franco-Paredes C. Inmunogenicidad y tolerancia de una vacuna contra influenza, en una población mexicana mayor de 55 años de edad. \\ Salud Publica Mex 2005;47:155-162.}

\begin{abstract}
Resumen
ObjetivosVerificar la inmuno genicidad y tolerancia de una vacuna purificada, inactivada y fraccionada contra influenza, en adultos mexicanos derechohabientes de Petróleos Mexicanos (Pemex). Material y métodos. Se incluyeron 90 adultos mayores de 55 años de edad, derechohabientes de los servicios médicos del Hospital Central Sur Pemex, durante los meses de noviembre y diciembre de 2000. Los criterios evaluados en relación con la inmunogenicidad incluyeron el porcentaje de sujetos protegidos, cuantificados por medio de anticuerpos antihemaglutininas superior o igual a 1:40, así como por el porcentaje de seroconversión determinado por el título inicial de anticuerpos multiplicado por un factor $4 X$. Los criterios secundarios fueron la frecuencia de reacciones adversas tanto locales como sistémicas. Se realizaron estudios de afinidad antígeno-anticuerpo para determinar la respuesta policlonal de anticuerpos y de anticuerpos de alta afinidad prevacunación y posvacunación. Se calcularon frecuencias y porcentajes. Resultados. Se identificó una seroprotección en $95.6 \%$ de los sujetos a la cepa H1N 1, de $98.9 \%$ a la cepa H3N 2 y de $100 \%$ a la cepa B/ Yamanashi. En cuanto a los porcentajes de seroconversión, se identificó un incremento 4X de 74.4 para la cepa H 1N 1, de 88.9 para la cepa H 3N 2, y de 82.2 para la cepa B / Yama-
\end{abstract}

\author{
Ayala-Montiel O, Mascareñas C, García-Hernández D, \\ Rendón-Muñiz J, López I, Montaño LF, \\ Zenteno I, Franco-Paredes C. \\ Immunogenicity and safety \\ of the influenza vaccine, in a population \\ older than 55-years in Mexico. \\ Salud Publica Mex 2005;47:155-162.
}

(1) Hospital Central Sur, Petróleos Mexicanos (Pemex). México, DF, México.

(2) Aventis Pasteur México. México, DF, México.

(3) Gerencia de Regulación y Desarrollo Médico, Pemex. México, DF, México.

(4) Instituto de Referencia y Diagnóstico Epidemiológicos (InD RE). México, DF, México.

(5) Departamento de Biología Celular, Instituto N acional de Cardiología. México, DF, México.

Fecha de recibido: 12 de abril de 2004 • Fecha de aprobado: 25 de enero de 2005

Solicitud de sobretiros: Dr. César 0 Mascareñas. Aventis Pasteur, México. Avenida Universidad N 0. 1738, colonia C oyoacán, 04000 México, DF, México. Correo electrónico: cesar.mascarenas@ aventis.com 
nashi. Un total de 18 individuos (20\%) presentaron reacciones locales, mientras que 17 (18.8\%) presentaron reacciones sistémicas a los cinco días posvacunación y nueve sujetos (10\%) a los 28 días. Las reacciones locales a los cinco días consistieron en dolor, en 10 individuos (11.1\%); enrojecimiento, en ocho $(8.8 \%)$, e induración, en seis $(6.6 \%)$. Malestar general, cefalea y fiebre se presentaron a los cinco días en $10,8.8$, y $0 \%$ de individuos, respectivamente, y en $4.4,6.6$, y $0 \%$, respectivamente, a los 28 días. Conclusiones Esta vacuna contra influenza demostró ser altamente inmunogénica en adultos mexicanos mayores de 55 años de edad. Se demostró también la producción de anticuerpos de alta afinidad contra el virus, posterior a la vacunación. A demás, se identificó una frecuencia de reacciones locales y sistémicas similares a las previamente reportadas. Estos resultados pueden ser extensivos a instituciones de salud que atienden principalmente a adultos mayores de 55 años de edad, para incrementar las tasas anuales de cobertura de vacunación contra influenza.

Palabras clave: influenza; inmunogenicidad; tolerancia; México nashi strain in $82.2 \%$. Eighteen (20\%) subjects developed local reactions; 17 (18.8\%) developed a systemic reaction post vaccination at day 5 and nine subjects (10\%) at day 28 . Local reactions consisted of pain in $10(11.1 \%)$ subjects, redness in 8 (8.8\%), and induration in $6(6.6 \%)$. General malaise, headache, and fever were identified in $10,8.8$, and $0 \%$ of subjects, respectively, at day 5 , and in $4.4,6.6$, and $0 \%$, respectively, at day 28. Conclusions. Influenza vaccine was highly immunogenic in a healthy Mexican adult population aged 55 years and older.The generation of high-affinity antibodies to the virus after vaccination was also demonstrated. Local and systemic adverse reactions to the vaccine identified in our study were similar to those in previous reports. The results of this study can be extrapolated to other health institutions serving this adult population to increase influenza vaccine coverage rates.

Key words: influenza; immunogenicity; tolerance; Mexico
$\mathrm{L}$ as infecciones respiratorias agudas son responsables de aproximadamente $13 \%$ de muertes por todas las causas en personas mayores de 55 años de edad. ${ }^{1-3}$ Entre las etiologías más frecuentemente asociadas los virus de la influenza son de las más importantes. ${ }^{4-6}$ En México, gracias al Sistema de Vigilancia Epidemiológica para Influenza se ha comenzado a determinar su impacto en este grupo etáreo. ${ }^{7}$ Existe una relación clara entre la influenza y un exceso de mortalidad atribuible directamente a esta infección. ${ }^{8,9}$ Además, existe una bien documentada relación entre la infección por influenza y un incremento en la morbilidad en adultos mayores con condiciones médicas de alto riesgo. ${ }^{10}$ En presencia de estas situaciones médicas existe un incremento de hasta cinco veces en las tasas de hospitalización asociadas a influenza. ${ }^{11,12}$

Las personas mayores de 55 años de edad, y particularmente los mayores de 65 años, se consideran entre los principales grupos de riesgo para sufrir las complicaciones graves de la infección por influenza. ${ }^{13,14}$ Estas incluyen la neumonía bacteriana secundaria o las exacerbaciones de enfermedades crónicas, que resultan tanto en la necesidad de hospitalización como en un riesgo elevado de muerte. ${ }^{14,15}$ Estudios observacionales realizados en varios países han identificado la efectividad de la vacunación contra la influenza para disminuir las tasas de hospitalización por neumonías y para disminuir el riesgo de mortalidad por todas las causas. ${ }^{16-24}$ Además, la vacunación contra la influenza en el ámbito comunitario ha revelado una disminución de la tasa de hospitalización por enfermedades pulmonares y de las exacerbaciones de insuficiencia cardiaca congestiva. ${ }^{20}$ Diversos brotes de influenza en asilos de ancianos e instituciones que atienden generalmente a poblaciones mayores de 55 años de edad han demostrado provocar una tasa de ataque de $60 \%$ y tasas de letalidad de entre 30 y $40 \% .{ }^{12-17}$ La vacunación contra influenza en este escenario es altamente efectiva para reducir enfermedad aguda respiratoria en 56\% (IC 95\% 39-68), neumonías en 53\% (IC 95\% 35-66\%), hospitalizaciones en 48\% (IC 95\% 28-65) y muerte en 68\% (IC 95\% 56-76). ${ }^{25}$ Por todos los aspectos anteriores el grupo de sujetos mayores de 55 años de edad es considerado como un grupo prioritario de vacunación contra la influenza.

En relación con la inmunogenicidad de la vacuna contra la influenza se piensa que los adultos mayores generalmente no responden eficientemente al estímulo antigénico provocado por la administración de la vacuna, debido probablemente a una respuesta humoral insuficiente. ${ }^{26-29}$ La vacuna contra la influenza está registrada en muchos países, en los cuales se emplea rutinariamente en grupos de alto riesgo y en los cuales se realizan ensayos para determinar su tolerancia e inmunogenicidad. ${ }^{10,13}$ Además, cada actualización de la composición de la vacuna es objeto de ensayos para evaluar la seguridad e inmunogenicidad de las nuevas cepas incluidas. ${ }^{10}$ Sin embargo, no ha habido hasta el momento estudios para evaluar la inmunogenicidad 
y tolerancia de la vacuna inactivada contra la influenza en poblaciones mexicanas. Asimismo, pocos estudios han evaluado estos parámetros en instituciones que atienden a poblaciones de adultos mayores, quienes se beneficiarían principalmente de políticas preventivas, como la vacunación contra la influenza.

El objetivo del presente estudio fue verificar la inmunogenicidad y la tolerancia de una vacuna contra la influenza, fórmula 2000-2001, comercializada en México desde 1997, en adultos mexicanos mayores de 55 años de edad, derechohabientes de los servicios médicos de Petróleos Mexicanos (Pemex).

\section{Material y métodos}

\section{Diseño del estudio}

El estudio consiste en un ensayo prospectivo, monocéntrico, no aleatorizado. Se incluyeron 90 adultos mayores de 55 años de edad derechohabientes de los servicios médicos del Hospital Central Sur Pemex, quienes acudieron al Departamento de Medicina Preventiva durante los meses de noviembre y diciembre de 2000. Todos cumplieron con los criterios de inclusión para el estudio, sujetos mayores de 55 años de edad, sin antecedentes de alergia a las proteínas de huevo y al timerosal; sin fiebre el día de la vacunación; sin estar recibiendo tratamientos que pudieran alterar la respuesta inmunitaria (corticoides, agentes anticancerosos, agentes inmunomoduladores). Se obtuvo el consentimiento informado de los sujetos participantes en el estudio, de acuerdo con lo que establecen las directrices de la International Conference on Harmonisation, por un lado, y las Buenas Prácticas Clínicas, de la Declaración de Helsinki, por el otro. Tanto el protocolo como el formato de consentimiento informado fueron aprobados por el Comité de Investigación y Etica del Hospital Central Sur Pemex. Posterior a firmar esta carta de consentimiento informado se procedió a aplicar la vacuna a los 90 sujetos del estudio.

Se utilizó la vacuna antigripal inactivada trivalente tipos A y B, Subvirión, Fórmula 2000-2001. La dosis de la vacuna $(0.5 \mathrm{ml})$ suplida en una jeringa prellenada contiene $15 \mu \mathrm{g}$ de hemaglutinina de cada una de las siguientes cepas virales: A/New Caledonia/20/99 (H1N1), A/Panamá/2007/99 (H3N2) y B/Yamanashi/ 166/98; gelatina a $0.05 \%$ como estabilizador y tiomersal a 1:10 000 como agente de conservación.

Los criterios evaluados en relación con la inmunogenicidad incluyeron el porcentaje de sujetos protegidos por la generación de títulos de anticuerpos anti-hemaglutininas superiores o iguales a 1:40. Asimismo, el porcentaje de seroconversión evaluado por el título inicial de anticuerpos, multiplicado por un factor igual de por lo menos 4 (4X). Las reacciones de inhibición de la hemaglutinación fueron desarrolladas por medio de métodos estándar de microtitulación después de la remoción de los inhibidores no específicos con enzimas destructoras de receptores. Muestras de suero pareado, pre y posvacunación del mismo sujeto fueron simultáneamente analizadas en duplicado para cada una de las tres cepas representadas en la vacuna. Los títulos de anticuerpos fueron expresados como el recíproco de la dilución más alta exhibiendo presencia de anticuerpo antihemaglutininas. Para el cálculo, un título de más de 5 fue arbitrariamente asignado al suero con un título $<10$. Un título $\geq 40$ fue considerado protector, de acuerdo con las prácticas recomendadas.

La determinación de anticuerpos anti-influenza en suero se realiza mediante la técnica de inhibición de la hemaglutinina en la cual se considera a los virus de referencia inactivados correspondientes a los de la formulación de la vacuna de la temporada, se estandarizan mediante la técnica de hemaglutinación y retrotitulación. Los sueros son previamente tratados y hemadsorbidos. La inhibición se lleva a cabo en placas de 96 pozos con fondo en $V$ en donde se diluyen los sueros y se agrega el virus estandarizado. Después de incubarlas y agregarles una suspensión de eritrocitos de pavo, se reporta en títulos de inhibición de la hemaglutinación. ${ }^{30,31}$

Los criterios secundarios incluyeron la frecuencia de aparición de reacciones adversas. Estas fueron determinadas por la proporción de sujetos con reacciones inmediatas, consideradas como aquellas que se presentaron durante los siguientes 30 minutos después de la vacunación; y por la proporción de sujetos con una reacción local o sistémica dentro de los primeros 28 días posteriores a la administración de la vacuna. Las reacciones locales comprendieron dolor, enrojecimiento e induración. El dolor fue clasificado en leve (ligero y bien tolerado), moderado (molesta al movimiento pero hay actividad conservada) y severo (impide actividad normal). El enrojecimiento, en leve $(<20 \mathrm{~mm})$, moderado (entre $20 \mathrm{~mm}$ y $30 \mathrm{~mm}$ ) y severo $(>30 \mathrm{~mm})$; el mismo criterio fue aplicado a la induración.

\section{Vacunación y seguimiento}

La vacuna antigripal fue inyectada por una enfermera sanitarista, vía intramuscular en la región deltoidea del brazo derecho. Se le solicitó al Departamento de Medicina Preventiva reportar cualquier evento de tipo inmediato relacionado con la vacunación que fuese 
grave o que provocara un riesgo para la vida del sujeto vacunado. A todos los sujetos se les entregó una hoja diaria de monitoreo con la cual acudieron a los cinco días de haber recibido la vacuna para una exploración médica. Las reacciones locales en el sitio de la inyección fueron reportadas en el autodiario de monitoreo de acuerdo con la percepción del vacunado y revisadas a los cinco días de la aplicación por los médicos investigadores. A los vacunados también se les solicitó medir la temperatura axilar y reportarla en el autodiario de monitoreo diariamente después de la vacunación si se percibían con elevación de la temperatura; esta información se revisó nuevamente a los 28 días después de la aplicación, en la última visita del vacunado.

\section{Estudios de afinidad antígeno-anticuerpo}

Se realizaron también estudios de afinidad antígenoanticuerpo con los sueros de los 90 sujetos del estudio. Se obtuvieron muestras prevacunación y posvacunación. El análisis se realizó utilizando una incubación de 18 horas en un ensayo en el cual se fijó el antígeno viral a la placa utilizando buffer de carbonatos, con la intención de obtener un valor elevado de anticuerpo, ya que se asumió que los pacientes incluidos en este estudio habían sido seleccionados con un parámetro que consideraba un historial clínico de no haber sufrido gripe o influenza en un periodo de cuando menos seis meses previos a la aplicación de la vacuna. También se determinó la presencia de anticuerpos de alta afinidad dirigidos contra hemaglutininas y neuraminidasa utilizando un ensayo similar con un periodo de incubación de una hora únicamente.

\section{A nálisis de los datos}

Para el análisis estadístico se calcularon las frecuencias y los porcentajes de los sujetos con reacciones locales y sistémicas, así como los porcentajes de la respuesta inmunogénica a la administración de la vacuna.

\section{Resultados}

Un total de 90 sujetos participaron en el estudio. De los participantes, 57 (63\%) pertenecieron al sexo femenino y $33(36 \%)$ al sexo masculino. La edad promedio fue de 69.7 años, con un intervalo de 55 a 95 años. En relación con los parámetros de inmunogenicidad se identificó una seroprotección con títulos de anticuerpos mayores a 1:40 posterior a la administración de la vacuna en $95.6 \%$ de los sujetos a la cepa H1N1, de $98.9 \%$ a la cepa H3N2 y de 100\% a la cepa B/Yamanashi, comparándose con $16.7,8.9$, y $15.5 \%$ de porcentajes basales prevacunación (figura 1). En cuanto a los porcentajes de seroconversión, cuantificados por un incremento $4 \mathrm{X}$, éstos fueron de $74.4 \%$ para la cepa $\mathrm{H} 1 \mathrm{~N} 1$, de $88.9 \%$ para la cepa H3N2, y de $82.2 \%$ para la cepa B/Yamanashi (figura 2).

Un total de 18 individuos (20\%) presentaron por lo menos un tipo de reacción local, mientras que 17 $(18.8 \%)$ presentaron reacciones sistémicas a los cinco días posvacunación y nueve sujetos (10\%) a los 28 días (figura 3). Las reacciones locales consistieron en dolor en el sitio de la aplicación a los cinco días posvacunación en 10 individuos (11.1\%), siendo leve en cinco $(5.6 \%)$ y moderado en otros cinco sujetos (5.6\%). Enrojecimiento en el sitio de administración se presentó en ocho sujetos $(8.8 \%)$, siendo leve en seis $(6.6 \%)$ y moderado en dos $(2.2 \%)$. La induración en el sitio de administración se encontró en seis sujetos $(6.6 \%)$, siendo leve en tres $(3.3 \%)$ y moderada en tres $(3.3 \%)$ (cuadro I). No se identificaron reacciones locales en ninguno de los individuos a los 28 días. No se presentaron reacciones inmediatas en ninguno de los sujetos vacunados.

Las reacciones sistémicas asociadas a la vacuna fueron determinadas a los 5 y 28 días posvacunación. Malestar general, cefalea y fiebre se presentaron a los cinco días posvacunación en 10, 8.8, y $0 \%$ de individuos, respectivamente. Estos mismos efectos fueron reportados a los 28 días posvacunación en $4.4,6.6$, y $0 \%$ de los sujetos, respectivamente.

En relación con los estudios de afinidad antígenoanticuerpo realizados en los sueros de estos pacientes,

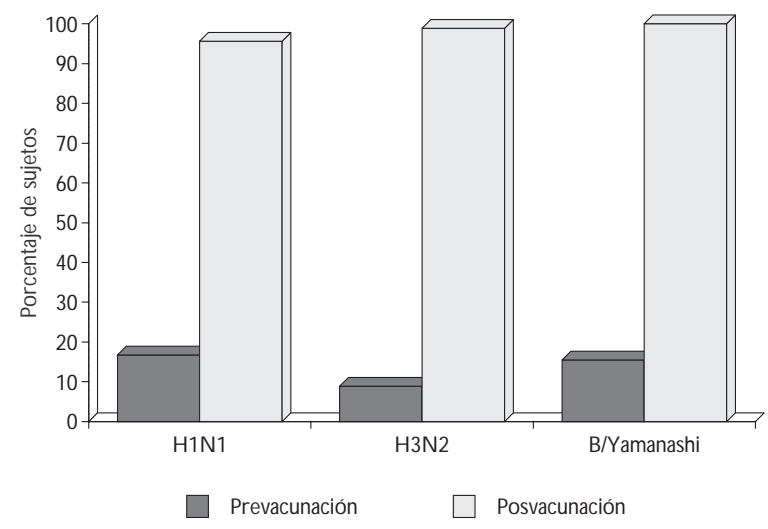

(Inducción de anticuerpos seroprotectores con titulación >1:40)

Figura 1. Porcentaje de protección posterior a la vacunación contra influenza. Hospital de PetróLeos Mexicanos. México, noviembre-diciembre 2000 


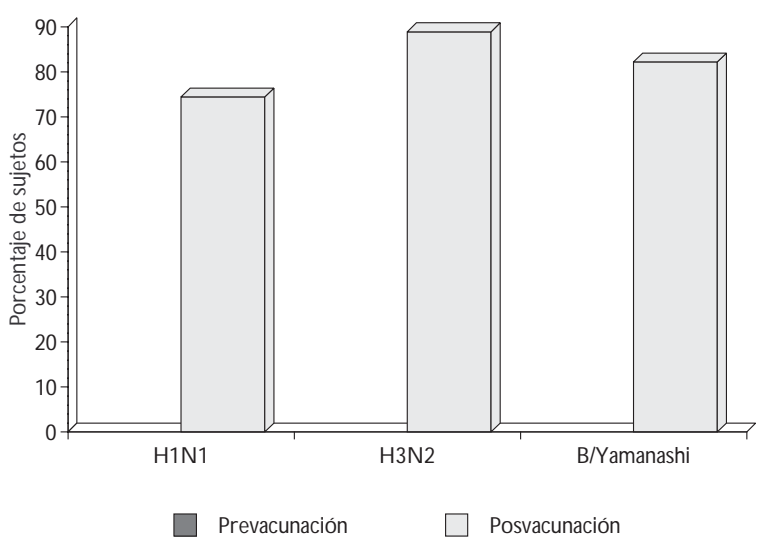

(Inducción de anticuerpos protectores con un incremento $4 \mathrm{X}$ )

Figura 2. Porcentaje de seroconversión posterior a LA ADMINISTRACIÓN DE LA VACUNA EN ESTUDIO CONTRA influenza, en sujetos del Hospital de Petróleos Mexicanos. México, noviembre-diciembre 2000

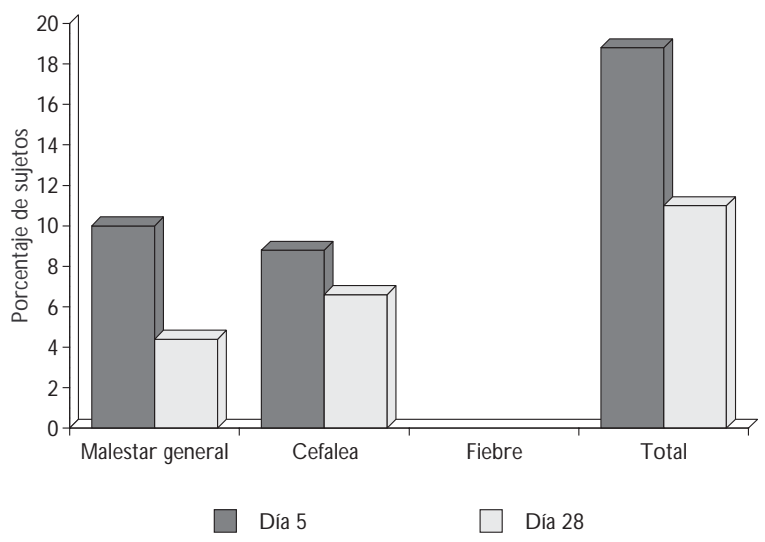

Figura 3. Porcentaje de Reacciones adversas sistéMICAS POSTERIOR A LA ADMINISTRACIÓN DE LA VACUNA EN estudio contra influenza, en SUJetos del Hospital de Petróleos Mexicanos. México, noviembre-diciemBRE 2000

se determinó el incremento promedio de alta y baja afinidad, el cual se refiere al diferencial obtenido entre la muestra basal y la final. Los resultados mostraron que existía una gran cantidad de anticuerpos en la muestra final. Se identificó que estos anticuerpos reaccionaron contra los componentes del virus, especialmente a las proteínas de la cápside. En la determinación de anticuerpos de alta afinidad que están dirigidos
Cuadro I

Porcentaje de reacciones locales en sujetos MAYORES DE 55 AÑOS DE EDAD, POSTERIOR A LA ADMINISTRACIÓN DE LA VACUNA EN ESTUDIO CONTRA influenza. Hospital de Petróleos Mexicanos. MéXICO, NOVIEMBRE-DICIEMBRE 2000

\begin{tabular}{|c|c|}
\hline Reacciones locales & $\begin{array}{c}\text { Vacuna en estudio }(n=90) \\
\text { Frecuencia }(\%)\end{array}$ \\
\hline Dolor & $10(11.1)$ \\
\hline Leve & $5(5.6)$ \\
\hline Moderado & $5(5.6)$ \\
\hline Severo & 0 \\
\hline Enrojecimiento & $8(8.8)$ \\
\hline Leve $(<20 \mathrm{~mm})$ & $6(6.6)$ \\
\hline Moderado (20-30mm) & $2(2.2)$ \\
\hline Severo (>30mm) & 0 \\
\hline Induración & $6(6.6)$ \\
\hline Leve $(<20 \mathrm{~mm})$ & $3(3.3)$ \\
\hline Moderada $(20-30 \mathrm{~mm})$ & $3(3.3)$ \\
\hline Severa (> 30mm) & 0 \\
\hline Total de reacciones locales & $24(26)$ \\
\hline Total de sujetos con reacciones locales & $18(20)$ \\
\hline
\end{tabular}

contra los antígenos inmunodominantes (hemaglutininas y neuraminidasas), se observó una importante generación de éstos posterior a la vacunación. La concentración de anticuerpos de alta afinidad fue menor a la concentración total de anticuerpos.

\section{Discusión}

La respuesta inmunogénica de la vacunación contra la influenza rara vez excede un año de protección. ${ }^{10,12}$ Los factores determinantes para obtener una mayor respuesta inmunogénica son la infección previa por una cepa cercanamente relacionada con la cepa infectante circulante o la historia de vacunación previa. ${ }^{1}$ No obstante, se ha observado que la inmunogenicidad asociada a la vacunación contra la influenza con vacunas inactivadas varía de acuerdo con la edad y estado de salud de los sujetos vacunados. La vacuna es efectiva en producir protección en más de $90 \%$ de adultos jóvenes sanos, sin embargo, en sólo 30 a 40\% de individuos mayores de 65 años de edad con enfermedades crónicas debilitantes. ${ }^{32}$

Los resultados demuestran que la vacuna estudiada contra la influenza es altamente inmunogénica en 
adultos mexicanos mayores de 55 años de edad. En este grupo las complicaciones y mortalidad asociadas a influenza son mayores y, por lo tanto, la vacunación resulta una estrategia preventiva fundamental. ${ }^{25,33,34} \mathrm{Un}$ meta-análisis publicado recientemente ha demostrado que la vacunación contra la influenza en pacientes mayores de 55 años de edad a escala poblacional reduce la tasa de hospitalización por neumonía e influenza en promedio 33\% (IC 95\% 27-38) y reduce también la mortalidad por todas las causas en 50\% (IC 95\% 45-56). ${ }^{35}$ Existe en México una alta tasa de hospitalización en adultos mayores ocasionada por infecciones de vías aéreas en hospitales de Pemex. La alta proporción de hospitalizaciones en adultos mayores en estos servicios médicos justifica la evaluación por parte de la institución de políticas preventivas de vacunación contra influenza.

La administración de la vacuna contra la influenza se estima que genera una respuesta protectora en 70 a $90 \%$ de los sujetos vacunados. ${ }^{32}$ Estas figuras generalmente se refieren en la literatura a sujetos sanos y en temporadas en las que la cepa circulante se encuentra cubierta por las cepas de la vacuna. Los resultados de nuestro estudio demuestran un elevado porcentaje de seroprotección y seroconversión generada por la vacuna. Además, en los ensayos de afinidad antígenoanticuerpo se demostró no únicamente la producción policlonal favorable de anticuerpos posvacunación específicos para el virus, sino también de anticuerpos de alta afinidad contra la hemaglutinina y la neuraminidasa virales. El hallazgo de una menor concentración de anticuerpos de alta afinidad, comparada con la concentración total de anticuerpos es un resultado esperado. ${ }^{36}$ Sin embargo, la identificación de los anticuerpos de alta afinidad es un hallazgo crítico en nuestro estudio, ya que éstos son los que entran en contacto con el virus en las fases primarias de la infección.

Aunque este estudio fue realizado en adultos mayores de 55 años de edad quienes acudieron al departamento de medicina preventiva y no por necesidad de servicios de atención clínica aguda, sus resultados son extrapoblables a adultos mayores mexicanos con enfermedades crónicas consideradas como de alto riesgo para desarrollar complicaciones por la influenza. A pesar de la variabilidad de la respuesta inmunogénica de la vacuna en pacientes con enfermedades crónicas como insuficiencia renal crónica, ${ }^{37-39}$ artritis reumatoide ${ }^{40}$ diabetes mellitus, ${ }^{32}$ enfermedad pulmonar obstructiva crónica, ${ }_{1}^{41,42}$ etcétera, existe un consenso mundial sobre la prioridad de la vacunación de estos grupos de pacientes.
Un hallazgo relevante de nuestro estudio es la presencia de una frecuencia de reacciones locales y sistémicas severas, similares a las notificadas en diversos estudios. ${ }^{43,44}$ Asimismo, identificamos una baja incidencia de reacciones locales y sistémicas leves o moderadas asociadas a la administración de la vacuna, las cuales son comparables a las reportadas en la literatura mundial. ${ }^{1,10,12}$ Estos hallazgos demuestran la adecuada seguridad y tolerancia de la vacunación contra la influenza en adultos mayores de 55 años de edad, en quienes en muchas ocasiones el temor a las reacciones de la vacunación son altamente prevalentes.

Diversos estudios realizados en los últimos 30 años que analizan los parámetros de costo-efectividad y costo-beneficio de la vacunación anual contra la influenza han demostrado importantes beneficios en salud poblacional y en aspectos económicos y de productividad. Estos beneficios incluyen una reducción de $72 \%$ en ausentismo laboral asociado a influenza, 15 a $43 \%$ relacionado con infecciones del tracto respiratorio superior y una disminución de ausentismo laboral por todas las causas. Asimismo, los resultados de estudios publicados recientemente han demostrado una dramática reducción de los costos directos e indirectos con la vacunación anual contra la influenza. Dentro de los costos directos se incluyen los costos de vacunación y los costos del tratamiento médico asociado al tratamiento de la infección y sus complicaciones. . $^{1045-46}$ Las personas vacunadas presentan un menor número de cuadros infecciosos de vías respiratorias, menos días de ausentismo laboral y tienen un significativo menor número de visitas al médico. Por esta razón, la vacunación anual contra la influenza representa una estrategia preventiva de prioridad nacional por sus efectos en salud y por tener efectos demostrados en estudios de análisis costo-beneficio y costo-efectividad. ${ }^{45,46}$

En conclusión, los resultados de este estudio demuestran la baja incidencia de reacciones adversas a la administración de la vacuna estudiada contra la influenza y la generación de una respuesta inmunogénica protectora en adultos mayores de 55 años de edad. La realización de este estudio en un hospital con una alta incidencia de hospitalización en este grupo sugiere que los resultados pueden hacerse extensivos a otras instituciones de salud con elevada morbilidad y mortalidad asociada a infecciones de vías aéreas. Resulta evidente la importancia de fortalecer en el ámbito nacional un incremento en la cobertura de la vacunación contra la influenza, dirigiéndose principalmente a las poblaciones de alto riesgo, como la de adultos mayores de 55 años de edad. 


\section{Referencias}

1. Pregliasco F, Mensi C, Serpilli W, Speccher L, Masella P, Belloni A. Immunogenicity and safety of three commercial influenza vaccines in institutionalized elderly. Aging C lin Exp Res 2001; 13: 38-43.

2. Monto AS. Influenza vaccines for the elderly. N Engl J Med 1994;331: 807-808.

3. N ichol KL, Margolis KL,W uorenna J,Von Stenberg T.The efficacy and cost-effectiveness of vaccination against influenza among elderly persons living in the community. N Engl J Med 1994; 331: 778-784. 4. Cate TR. Clinical manifestations and consequences of influenza. Am J Med 1987; 82 (SS6A): 15-19.

5. Glezen W P. Serious morbidity and mortality associated with influenza epidemics. Epidemiol Rev 1993; 4: 25-44.

6. Kohn RP. Cause of death in very old people. JAMA 1982; 247: 2793-

2797.

7. Sistema $\mathrm{N}$ acional de Vigilancia Epidemiológica. Boletín Epidemiológico 1999;16(44): Semana 44.

8. Barker W H, Mullooly JP. Influenza vaccination of elderly persons: Reduction in pneumonia and influenza hospitalizations and death. JAMA 1980; 244: 2547-2549.

9. N ichol KL.The efficacy, effectiveness and cost-effectiveness of inactivated influenza virus vaccines. Vaccine 2003; 21:1769-1775. 10. Centers for D isease Control and Prevention. Prevention and control of influenza. Recommendations of the Advisory Committee on Immunization Practices. MMW R 2004; 53(RR-6): 1-40.

11. McBean AM, Babish JD,W arren JL. The impact and cost of influenza in the elderly.Arch Intern Med 1993; 153: 2105-2111.

12. Hak E, N ordin J,W ei F, Mullooly J, Poblete S, Strikas R et al. Influence of high-risk medical conditions on the effectiveness of influenza vaccination among elderly members of 3 large managed-care organizations. C lin Infect D is 2002; 35: 370-377.

13. Centers for Disease Control and Prevention. Prevention and control influenza. Recommendations of the Advisory Committee on Immunizations Practices (ACIP). MMW R 2003; 52 (RR-8): 1-34. 14. Scheiblauer $H$, Reinacher M, Tashiro M, Rott R. Interactions between bacteria and influenza. A virus in the development of influenza pneumonia.J Infect D is 1992; 166: 783-791.

15. Schwarzmann SW, Adler JL, Sullivan RF Jr, Marine W M. Bacterial pneumonia during the Hong Kong influenza epidemic of 1968-1969. Arch Intern Med 1971; 127: 1037-1041.

16. Govaert TM,Thijs CT, Masurel N, Sprenger MJ, Dinant GJ, Knottnerus JA.The efficacy of influenza vaccination in elderly individuals. A randomized double-blind placebo-controlled trial. JAMA 1994;272: 1661-1665

17. Mulloolly JP, Bennet MD, Hornbrook MC, Barker W H, W illiams W W, Patriarca PA et al. Influenza vaccination programs for elderly persons: Cost-effectiveness in a health maintenance organization. Ann Intern Med 1994; 121: 947-952.

18. Foster DA, Talsma A, Furumoto-D awson A, 0 hmit SE, Margulies JR, Arden $\mathrm{N} \mathrm{H}$ et al. Influenza vaccine effectiveness in preventing hospitalization for pneumonia in the elderly. Am J Epidemiol 1992; 136: 296-307.

19. 0 hmit SE, Monto AS. Influenza vaccine effectiveness in preventing hospitalizations among the elderly during influenza type $A$ and type $B$ seasons. Int J Epidemiol 1995; 24: 1240-1248.

20. N ichol KL,W uornema J, von SternbergT. Benefits of influenza vaccination for low-, intermediate, and high-risk senior citizens. Arch Intern Med 1998; 158: 1769-1776.
21.A hmed AE, N icholson KG, N guyen-Van-Tam JS, Pearson JC. Effectiveness of influenza vaccination in reducing hospital admissions during the epidemic of 1989-1990. Epidemiol Infect 1997; 118:27-33. 22. A hmed AE, N icholson KG, N guyen-Van-Tam JS. Reduction in mortality associated with influenza vaccine during 1989-1990 epidemic. Lancet 1995; 346: 591-595.

23. Stamboulian D, Bonvehi PE, N acinovich FM, Ruttimann RW. Immunization against influenza in the elderly: The Argentinean experience, 1993-1997.Vaccine 1999; 17 Suppl 1:S53-S56.

24. W ang CS,W ant ST, Chou P. Efficacy and cost-effectiveness of influenza vaccination of the elderly in a densely populated and unvaccinated community. Vaccine 2002; 20: 2494-2499.

25. G ross PA, Hermogenes AW, Sacks HS, Lau J, Levandowski RA.The efficacy of influenza vaccine in elderly persons:A meta-analysis and review of the literature.Ann Intern Med 1995; 123:518-527.

26. LaM ontagne JR, N oble GR, Q uinnan GV, C urlin GT, Blackwelder W C Smith JL et al. Summary of clinical trials of inactivated influenza vaccine1978. Rev Infect Dis 1983; 5: 723-736.

27. Cate TR, Coudh RB, Parker D, Baxter B. Reactogenicity, immunogenicity, and antibody persistence in adults given inactivated influenza virus vaccines - 1978. Rev Infect D is 1983; 5: 737-747. 28. Beyer W E, Palache AM, Baljet M, Masurel N . Antibody induction by influenza vaccines in the elderly:A review of the literature.Vaccine 1989; 7(5):385-394.

29. Fagiolo U,Amadori A, Cozzi E, Bendo R, Lama M, D ouglas A et al. Humoral and cellular immune response to influenza virus vaccination in aged humans. Aging 1993; 5: 451-458.

30. Panamerican Health $\mathrm{O}$ rganization, W ord Health 0 rganization, Centers for Disease Control and Prevention. Diagnosis of influenza and other respiratory viruses. Course Manual;A pril 21-25; Santiago, C hile; 1997.

31. Panamerican Health O rganization, Centers for Disease Control. Serologic diagnosis of influenza viruses infections by hemaglutination inhibition. Course Manual; 2004 May 03-07; Atlanta, Georgia, EUA. 32. Centers for Disease Control and Prevention. Epidemiology and prevention of vaccine-preventable diseases. Seventh Edition. Atlanta (GA): CDC; 2002: 190-204

33. N ichol KL, Goodman M. Cost-effectiveness of influenza vaccination for healthy persons between ages 65 and 74 years. Vaccine 2002;20 Suppl 2:S21-S24.

34. N ichol KL. C linical effectiveness and cost-effectiveness of influenza vaccination among healthy working adults. Vaccine 1999; 17 Suppl 1:S67S73.

35.Vu T, Farish S, Jenkins M, Kelly H. A meta-analysis of effectiveness of influenza vaccine in persons aged 65 years and over living in the community.Vaccine 2002; 20(13-14):1831-1836.

36. Treanor J. Influenza virus. En: Mandell GL, Bennett SE, 0 din R, ed. D ouglas, and Bennett's. Principles and practice of infectious diseases. $5^{\text {th }}$ Ed. N ueva York (N Y): C hurchill Livingstone; 2000: 1823-1849.

37. Pabico RC, D ouglas RG, Betts RF, McKenna BA, Freeman RB. Influenza vaccination of patients with glomerular disease: Effects on creatinine clearance, urinary protein excretion, and antibody response. Ann Intern Med 1974; 81: 171-174.

38. 0 sanloo EO, Berlin BS, Popli S, Ing TS, Cummings JE, G andhi VC et al. Antibody responses to influenza vaccination in patients with chronic renal failure. Kidney Int 1978; 14: 614-618.

39. Jordan MC, Rousseau W E, Tegtmeier GE, N oble GR, Muth RG, Chin $T D$. Immunogenicity of inactivated influenza virus vaccine in chronic renal failure.Ann Intern Med 1973; 14:614-618.

40. Chalmers A, Scheifele D, Patterson C, W illiams D, W eber J, Shuckett $R$ et al. Immunization of patients with rheumatoid arthritis against 
influenza:A study of vaccine safety and immunogenicity. J Rheumatol 1994; 21: 1203-1206.

41. Ruben FL. Prevention and control of influenza: Role of vaccine.Am J Med 1987; 82(6A): 31-34.

42. Kubiet MA, González-Rothi RJ, Cottey R, Bender BS. Serum antibody response to influenza vaccine in pulmonary patients receiving corticosteroids. Chest 1996; 110: 367-370.

43. $\mathrm{N}$ ichol KL, Lind A, Margo lis KL, Murdoch M, McFadden R, Hauge M et al.The effectiveness of vaccination against influenza in healthy, working adults. N Engl J Med 1995; 333(14): 891-895.
44. Spila-Alegiani S, Salmaso S, Rota MC, Tossi AE, Raschetti R. Reactogenicity in the elderly of nine commercial influenza vaccines: Results from the Italian SVEVA Study.Vaccine 1999; 17: 1898-1904.

45. Szucs TD. Medical economics in the field of influenza- Past, present, and future.Virus Res 2004; 103(1-2): 25-30.

46. N ichol KL, Mendelman PL. Influence of clinical case definitions with differing levels of sensitivity and specificity on estimates of the relative and absolute health benefits of influenza vaccines among healthy adult workers and implications for economic analyses.Virus Res 2004; 103(12): 3-8. 